\title{
Oral ingestion of lidocaine for suicide: A case report
}

\section{Maryam Adimolmasali', Ali Teimouri², Hamid Reza Mokhtari', Mahdi Foroughian', Parvin Zohoorian Sadr', Mohammad Hossien Kamaloddini ${ }^{3^{*}}$}

'Department of Emergency Medicine, Faculty of Medicine, Mashhad University of Medical Sciences, Mashhad, Iran ${ }^{2}$ Student Research Committee, Mashhad University of Medical Sciences, Mashhad, Iran

${ }^{3}$ Clinical Toxicology Department, Imam Reza Hospital, Mashhad, Iran

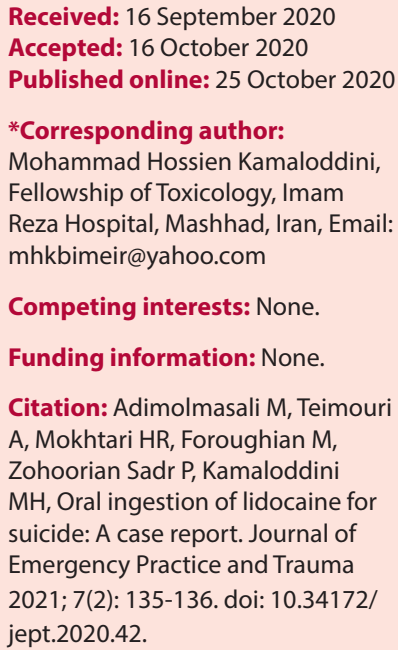

\begin{abstract}
Objective: Systemic toxemia with topical anesthetics could be fatal due to the use of anesthetics in various ways (oral, intravenous, intramuscular, or subcutaneous). Nowadays, topical anesthetics are used in inpatient and outpatient treatments. Despite its widespread usage, many physicians are not fully aware of the deadly side effects of lidocaine poisoning and its treatment. The objective of this case report is to highlight the diagnosis and treatment of lidocaine toxicity.

Case Presentation: A 21-year-old woman with a history of multiple suicide attempts ingested approximately three $10 \% \mathrm{w} / \mathrm{w}$ lidocaine sprays. The patient was brought to the emergency department of Imam Reza hospital of Mashhad University of Medical Science with cardiac arrest and respiratory failure. After successful cardiopulmonary resuscitation (CPR), she was admitted to the intensive care unit (ICU). She stayed in ICU for 2 days and we administered dobutamine drip and intravenous lipid emulsion for her then she was transferred to the general ward. We discharged her after 8 days.

Conclusion: Using BCLS and ACLS in case of cardiac arrest and administration of Lipid Emulsion with proper dosage are recommended treatments for lidocaine toxicity. Keywords: Lidocaine poisoning, Oral ingestion, Suicide
\end{abstract}

\section{Introduction}

Lidocaine is used for local anesthesia, antiarrhythmic, controlling interstitial cystitis (bladder pain syndrome) and seizure $(1,2)$. It is available in various forms including infusion and injection solution, patch, gel, ointment, liquid and spray (3). Lidocaine is used in a wide range of cases, from outpatients to inpatients and also in different facilities. from clinics and emergency departments to operation rooms (3).

The mechanism of lidocaine in local anesthesia is inhibition of sodium channel protein in the neuron membrane and stabilization of neuron membrane (4). Toxicity of lidocaine includes central nervous system (CNS) toxicity that identifies as numbness, tinnitus, generalized convulsions, unconsciousness, and cardiovascular system toxicity (that identifie as hypotension, arrhythmia, and bradycardia) (5).

In this case report we introduced a patient who attempted suicide by using lidocaine orally which is rare and noticeable. The patient experienced severer systemic side effects of lidocaine toxicity. In this study we aim to make emergency physicians aware of the clinical manifestations and treatment of lidocaine toxicity.

\section{Case Presentation}

Our patient was a 21-year-old woman with a history of the neuropsychiatric disorder and multiple suicide attempts. She ingested three $10 \% \mathrm{w} / \mathrm{w}$ lidocaine sprays.

Her signs and symptoms initiated with restlessness, agitation, and palpitations and after several hours she had a cardiac arrest. Our patient was brought to the emergency department of Imam Reza hospital of Mashhad University of Medical Sciences for cardiac arrest and respiratory failure. After successful cardiopulmonary resuscitation (CPR) and intubation with appropriate ventilation (12 ventilation per minute), the patient was sent to the intensive care unit (ICU). At the time of admission in ICU, the heart rate was 96 and blood pressure was 70/100.

In physical examination, the patient had no fever and she had bilateral Babinski sign in plantar reflux and normal pupil size. In laboratory investigation, complete blood count, electrolytes, renal function test (creatinine and urea) and liver function test were within normal ranges. Also, troponin test was negative. On bedside echocardiography, ejection fraction (EF) was reported about $20 \%$ with no valvular abnormalities at the time of admission.

Chest X-ray showed evidence of pulmonary edema on both sides and involvement of opacity in the right lung. During ICU admission, she had cardiac arrest two times. Fortunately, our CPR with advanced cardiac life support (ACLS) protocol was resuscitative. 
We administered lipid emulsion therapy with $20 \%$ lipid emulsion with a dosage of $1.5 \mathrm{~mL} / \mathrm{kg}$ IV over 1 minute as a bolus and $0.25 \mathrm{~mL} / \mathrm{kg} / \mathrm{minute}$ as a continuous infusion. We also started dobutamine drip with a dosage of $1.5 \mathrm{mcg} /$ $\mathrm{kg} / \mathrm{min}$ IV continuous infusion. After 2 days, the patient was extubated, dobutamine infusion and lipid emulsion were discontinued and she was transferred to the general ward. On repeated bedside echocardiography in general ward, EF was improved to around 50\%. During the hospitalization period, the patient suffered from restless and agitation, thus, we administered quetiapine $(100 \mathrm{mg} / \mathrm{d}$ orally). She underwent psychiatric consultation before discharge. The patient was discharged from the hospital after 10 days with psychiatric follow-up.

\section{Discussion}

Lidocaine is a local anesthetic and a member of class $1 b$ antiarrhythmic drug. It is one of the most common local anesthetic drugs and is used in the treatment of ventricular arrhythmias (6).

Lidocaine toxicity may occur unintentionally or deliberately for suicidal purposes (5).

Iatrogenic lidocaine toxicity may occur due to wrong dose calculation, direct injection of the drug into the vein or artery and repeated dosing. Rate of severe systemic lidocaine toxicity is 1:10000 for epidurals and up to 1:2000 for peripheral nerve blocks $(7,8)$.

Lidocaine inhibits sodium channels and for this reason lidocaine toxicity has more effect on the cardiac system and CNS (7). CNS toxicity includes circumoral numbness, facial tingling, restlessness, vertigo, tinnitus, slurred speech, and tonic-clonic seizures (1).

Cardiac manifestations of lidocaine toxicity include prolonged QRS interval, hypotension, bradycardia, shock, and dysrhythmias (5). The recommended maximum dose for subcutaneous infiltration of lidocaine without epinephrine is $4.5 \mathrm{mg} / \mathrm{kg}$ and for lidocaine with epinephrine is $7 \mathrm{mg} / \mathrm{kg}$ (5). Asystole may occur in patients who take $800-1000 \mathrm{mg}$ of intravenous lidocaine. Our patient's weight was $73 \mathrm{~kg}$ and she received $300 \mathrm{~mL}$ lidocaine $10 \% \mathrm{w} / \mathrm{w}$ (totally $30000 \mathrm{mg}$ ) but we do not know about the amount of absorption. Serum levels of lidocaine are not readily available and knowing about this that does not help us about the management and treatment. Thus, lidocaine toxicity is diagnosed based on clinical manifestations and medical history (9). Sometimes computerized tomography (CT) or magnetic resonance imaging (MRI) may help to rule out other causes of transient neurologic symptoms.

Treatment of lidocaine toxicity is based on two principles:

First, management of cardiac arrhythmias should be based on basic and advanced cardiac life support (BCLS and ACLS), avoid using vasopressin, calcium channel blockers, beta-blockers, or local anesthetic (5). Second, administering lipid emulsion (20\%) with a dosage of 1.5 $\mathrm{mL} / \mathrm{kg}$ over one minute as a bolus and $0.25 \mathrm{~mL} / \mathrm{kg} / \mathrm{min}$ as a continuous infusion (5).
If heart failure persists, the dose of infusion can be doubled to $0.5 \mathrm{cc} / \mathrm{kg} / \mathrm{min}$. It is recommended that infusion should be continued for at least 10 minutes to improve cardiac instability (5). Rapid administration of lipid emulsion results in a good reversal of circulation and improved cardiac function by increasing EF. In our patient, EF increased for $20 \%$ to $50 \%$.

If seizure occurs, benzodiazepine is recommended (5).

\section{Conclusion}

Systemic toxemia with lidocaine could be fetal. Prompt diagnosis of its clinical signs is the key factor to prevent death. Emergency physicians and physician must be able to quickly identify lidocaine poisoning. Using BCLS and ACLS in case of cardiac arrest and administration of Lipid Emulsion with proper dosage are recommended treatments for lidocaine toxicity.

\section{Authors' contributions}

All authors contributed equally to this manuscript. all authors read and approved final manuscript.

\section{Ethical issues}

The patients signed informed consent containing the agreement to the publication of this case report and images.

\section{References}

1. DeToledo JC. Lidocaine and seizures. Ther Drug Monit 2000; 22(3): 320-2. doi: 10.1097/00007691-20000600000014 .

2. Nickel JC, Jain P, Shore N, Anderson J, Giesing D, Lee $\mathrm{H}$, et al. Continuous intravesical lidocaine treatment for interstitial cystitis/bladder pain syndrome: safety and efficacy of a new drug delivery device. Sci Transl Med 2012; 4(143): 143ra00. doi: 10.1126/scitranslmed.3003804.

3. Baselt RC. Disposition of Toxic Drugs \& Chemicals in Man. Biomedical Publications; 2017.

4. Muroi Y, Chanda B. Local anesthetics disrupt energetic coupling between the voltage-sensing segments of a sodium channel. J Gen Physiol 2009; 133(1): 1-15. doi: 10.1085/ jgp.200810103.

5. Sekimoto K, Tobe M, Saito S. Local anesthetic toxicity: acute and chronic management. Acute Med Surg 2017; 4(2): 152-60. doi: 10.1002/ams2.265.

6. Gangadharan S, Nawathe P, Schleien CL. 40 Cardiopulmonary resuscitation. In: Coté CJ, Lerman J, Anderson BJ, eds. A Practice of Anesthesia for Infants and Children. 6th ed. Philadelphia: Content Repository Only!; 2019. p. 908-20.e4. doi: 10.1016/b978-0-323-42974$0.00040-9$.

7. Dokken K, Fairley P. Sodium Channel Blocker Toxicity. Treasure Island, FL: StatPearls Publishing; 2020

8. Lee JT, Sanderson CR, Xuan W, Agar M. Lidocaine for cancer pain in adults: a systematic review and metaanalysis. J Palliat Med 2019; 22(3): 326-34. doi: 10.1089/ jpm.2018.0257.

9. Centini F, Fiore C, Riezzo I, Rossi G, Fineschi V. Suicide due to oral ingestion of lidocaine: a case report and review of the literature. Forensic Sci Int 2007; 171(1): 57-62. doi: 10.1016/j.forsciint.2006.05.003. 